\title{
The Effects of Hemodialysis on Tear Osmolarity
}

\author{
Muhittin Taskapili, ${ }^{1}$ Kubra Serefoglu Cabuk, ${ }^{2}$ Rukiye Aydin, ${ }^{3}$ Kursat Atalay, ${ }^{2}$ \\ Ahmet Kirgiz, ${ }^{2}$ Dede Sit, ${ }^{4}$ and Hasan Kayabasi ${ }^{4}$
}

\author{
${ }^{1}$ Prof. Dr. N. Resat Belger Beyoglu Eye Training and Research Hospital, 34421 Istanbul, Turkey \\ ${ }^{2}$ Ophthalmology Department, Bagcilar Training and Research Hospital, 34200 Istanbul, Turkey \\ ${ }^{3}$ Ophthalmology Department, Medipol University, 34214 Istanbul, Turkey \\ ${ }^{4}$ Nephrology Department, Bagcilar Training and Research Hospital, 34200 Istanbul, Turkey
}

Correspondence should be addressed to Kubra Serefoglu Cabuk; drqubra@gmail.com

Received 6 September 2015; Revised 21 October 2015; Accepted 26 October 2015

Academic Editor: Lei Zhou

\begin{abstract}
Copyright ( $(2015$ Muhittin Taskapili et al. This is an open access article distributed under the Creative Commons Attribution License, which permits unrestricted use, distribution, and reproduction in any medium, provided the original work is properly cited.
\end{abstract}

\begin{abstract}
Aim. To determine the effects of hemodialysis (HD) on tear osmolarity and to define the blood biochemical tests correlating with tear osmolarity among patients with end stage renal disease (ESRD). Material-Method. Tear osmolarity of ESRD patients before and after the hemodialysis program was determined as well as the blood biochemical data including glucose, sodium, potassium, calcium, urea, and creatinine levels. Results. Totally 43 eyes of 43 patients ( 20 females and 23 males) with a mean age of $53.98 \pm 18.06$ years were included in the study. Tear osmolarity of patients was statistically significantly decreased after hemodialysis ( $314.06 \pm$ 17.77 versus $301.88 \pm 15.22 \mathrm{mOsm} / \mathrm{L}, p=0.0001)$. In correlation analysis, pre-HD tear osmolarity was negatively correlated with pre-HD blood creatinine level $(r=-0.366, p=0.016)$. Post-HD tear osmolarity was statistically significantly correlated with the post-HD glucose levels $(r=0.305 p=0.047)$. Tear osmolarity alteration by HD was negatively correlated with creatinine alteration, body weight alteration, and ultrafiltration $(r=-0.426, p=0.004 ; r=-0.365, p=0.016$; and $r=-0.320, p=0.036$, resp.). There was no correlation between tear osmolarity and Kt/V and URR values. Conclusion. HD effectively decreases tear osmolarity to normal values and corrects the volume and composition of the ocular fluid transiently. Tear osmolarity alteration induced by HD is correlated with body weight changes, creatinine alterations, and ultrafiltration.
\end{abstract}

\section{Introduction}

Hemodialysis (HD) is the main treatment method in patients with end stage renal disease (ESRD) to correct the composition and volume of body fluids. The adequacy of HD is still a main subject for active investigation $[1,2]$.

There are some parameters present for evaluation of adequacy of HD such as $K t / V$ (a number used to quantify hemodialysis treatment adequacy, in which $K$ is the dialyzer clearance of urea, $t$ is the dialysis time, and $V$ is the volume of distribution of urea) and urea reduction ratio (URR) [3].

HD may alter the volume and composition of ocular fluid as well as the systemic hemodynamic parameters [4]. Presence of dry eye in patients with ESRD is known for years [5-7].

Dry eye disease is an important and common public health problem with $5-35 \%$ prevalence in general population, as it causes discomfort and deterioration in quality of vision [8]. Although, a direct method is still not present, dry eye questionnaires, Schirmer's test, and tear break-up time (TBUT) are in clinical use to support the dry eye diagnosis. Nowadays, tear osmolarity measurement is regarded as the most accurate way of diagnosis of dry eye disease [9]. As previous studies showed, reduction in aqueous tear flow as a result of lacrimal failure with or without accelerated evaporation from the tear film is major determinants of the tear hyperosmolarity $[8,10]$. Additionally, the electrolytes of the aqueous phase of the tear film can effect tear osmolarity [11]. Of the electrolytes present in the tear film, cations sodium and potassium (120-170 $\mathrm{mmol} / \mathrm{kg}$ and $6-42 \mathrm{mmol} / \mathrm{kg}$, resp.) and anions chloride and bicarbonate $(106-135 \mathrm{mmol} / \mathrm{kg}$ and $26 \mathrm{mmol} / \mathrm{kg}$, resp.) are the major contributors to tear osmolarity [12]. Measurement of tear osmolarity in clinical setting 
is easy with recently developed lab-on-a-chip technology, namely, TearLab (TearLab Corporation, San Diego, CA, USA), with $72.8 \%$ sensitivity and $92.0 \%$ specificity at a cutoff value of $312 \mathrm{mOsms} / \mathrm{L}$ [9].

In this study, we aimed to determine the effects of $\mathrm{HD}$, performed with isovolemic and standard sodium $\left(\mathrm{Na}^{+}\right)(138 \mathrm{mEq} / \mathrm{L})$ and potassium $\left(\mathrm{K}^{+}\right)(2 \mathrm{Eq} / \mathrm{L})$ containing dialysates, on tear osmolarity and to evaluate the correlation between blood biochemical tests and tear osmolarity in patients with ESRD.

\section{Material and Method}

2.1. Patients. Tear osmolarity of 43 eyes of 43 patients under the regular, 3 times per week, hemodialysis program in Bagcilar Education and Research Hospital, Hemodialysis Unit, was evaluated and McMonnies and Ho questionnaire was filled in between April 2014 and June 2014. The blood samples were taken and tear osmolarity was detected one minute before the beginning of the hemodialysis and 30 minutes after the termination of hemodialysis program. Patients with diabetic retinopathy and any rheumatic and connective tissue diseases, patients using any type of eye drops and wearing contact lenses, and patients with the history of ocular surgery were excluded from the study.

The study protocol was approved by the local ethics committee in Bagcilar Education and Training Hospital, Turkey. Informed consent was obtained from all subjects.

2.2. Laboratory Tests. Pre-HD blood samples were taken and tear osmolarity was detected one minute before the beginning of HD. The rate of diffusion and blood flow between body compartments reduce the effective $K$ and therefore $K t / V$ and result in the postdialysis rebound. To take account of these factors, $K t / V$ should ideally be calculated using a postdialysis sample taken 30-60 minutes after dialysis when the urea concentrations have reequilibrated [13]. As urea enters into tear fluid by simple diffusion [14], post-HD tear osmolarity was detected and biochemical samples were taken 30 minutes after the termination of HD session. Body weights of participants were recorded as well as the ultrafiltration amount. Serum urea, creatinine, glucose, sodium, potassium, calcium, and bicarbonate $\left(\mathrm{HCO}_{3}{ }^{-}\right)$levels were studied using the standard methods recommended by the manufacturer. Serum osmolarity was calculated with the formula [15]:

$$
\begin{aligned}
\text { Serum osmolarity }= & 2\left[\mathrm{Na}^{+}\right]+\frac{[\text { Glucose }]}{18} \\
& +\frac{[\text { Blood urea nitrogen }]}{2.8} .
\end{aligned}
$$

The urea reduction ratio (URR) is a number used to quantify dialysis treatment adequacy and similarly $\mathrm{Kt} / \mathrm{V}$ is also a number used to quantify hemodialysis treatment adequacy, in which $K$ is the dialyzer clearance of urea, $t$ is the dialysis time, and $V$ is the volume of distribution of urea.
TABLE 1: Clinical characteristics of the patients.

\begin{tabular}{lc}
\hline Gender (F/M) & $20 / 23$ \\
Age (years) & $53.98 \pm 18,06$ \\
Time of dialysis (years) & $5.12 \pm 5.67$ \\
Presence of hypertension & $32(76 \%)$ \\
Presence of diabetes mellitus & $16(38 \%)$ \\
Mean URR (\%) & $73.52 \pm 6.1$ \\
Mean spKt/V & $1.42 \pm 0.16$ \\
\hline
\end{tabular}

URR was calculated as a percentage of post-HD blood urea nitrogen (BUN) divided by pre-HD BUN. The singlepool $K t / V$ delivered by hemodialysis was estimated by the second-generation Daugirdas equation [16].

We have recorded the URR and $K t / V$ and investigated their associations with tear osmolarity.

Dry eye symptoms of the patients are evaluated with McMonnies and Ho questionnaire. Any score over 14.5 indicates a strong likelihood of dry eye disease [17]. Tear osmolarity was measured using lab-on-a-chip technology TearLab Osmolarity System (TearLab Corporation, 9980 Huennekens Street, Ste 100, San Diego, CA 92121, 1-855-8327522, USA), one minute before the beginning of HD and 30 minutes after the termination of HD. The measurements were performed at a stable room temperature of $25-25.5^{\circ} \mathrm{C}$ and the room humidity was 50-55\%. Quality control procedures were applied at the beginning of each day of patient testing by using reusable electronic check cards (provided by the manufacturer as a procedural quality control) to confirm the function and calibration of the TearLab Osmolarity System. A tear sample, approximately $50 \mathrm{~nL}$, was collected from the inferior lateral tear meniscus of the ocular surface by the same investigator.

2.3. Statistical Analysis. Statistical analysis was performed using Statistical Package for Social Sciences (SPSS, SPSS Inc., Chicago, IL, USA) version 21.0. Descriptive statistics were summarized as mean \pm SD or percentage. Paired samples $t$ test was performed in comparison of pre-HD and post-HD results. Chi square test was used in comparison of two groups. Pearson's correlation analysis was performed to determine the correlations of laboratory data with tear osmolarity. A $p$ value of $<0.05$ was regarded as statistically significant.

\section{Results}

Totally, 43 eyes of 43 patients (20 females and 23 males) with a mean age of $53.98 \pm 18.06$ years were included in the study.

McMonnies and Ho questionnaire was performed before $\mathrm{HD}$ to 41 patients because 2 patients were unable to complete the questionnaire. Mean questionnaire score was $6.27 \pm 5.02$. And all patients gave the same answers to the questionnaire after HD. There was only one patient who had dry eye according to the McMonnies and Ho questionnaire.

Descriptive characteristics of study participants are summarized in Table 1.

In the 37 of the 43 patients (86\%) URR was over $65 \%$ and $K t / V$ was over 1.2 and $\mathrm{HD}$ was adequate according to these two parameters. 
TABLE 2: The laboratory data analysis of patients before and after HD.

\begin{tabular}{lccc}
\hline & Before HD & After HD & $p$ \\
\hline Body weight & $70.59 \pm 15.95$ & $68.54 \pm 15.57$ & $\mathbf{0 . 0 0 0 1}$ \\
Tear osmolarity $(\mathrm{mOsm} / \mathrm{L})$ & $314.05 \pm 17.77$ & $301.88 \pm 15.22$ & $\mathbf{0 . 0 0 0 1}$ \\
Serum glucose $(\mathrm{mg} / \mathrm{dL})$ & $128.63 \pm 92.26$ & $121.77 \pm 42.66$ & 0.533 \\
Serum urea $(\mathrm{mg} / \mathrm{dL})$ & $130.96 \pm 31.03$ & $34.88 \pm 12.4$ & $\mathbf{0 . 0 0 0 1}$ \\
Serum creatinine $(\mathrm{mg} / \mathrm{dL})$ & $8.08 \pm 2.36$ & $2.87 \pm 1.01$ & $\mathbf{0 . 0 0 0 1}$ \\
Serum calcium $(\mathrm{mEq} / \mathrm{L})$ & $8.79 \pm 1,16$ & $9.43 \pm 0.71$ & $\mathbf{0 . 0 0 0 1}$ \\
Serum sodium $(\mathrm{mEq} / \mathrm{L})$ & $137.28 \pm 3.73$ & $136.47 \pm 2.69$ & 0.165 \\
Serum potassium $(\mathrm{mEq} / \mathrm{L})$ & $5.22 \pm 0.79$ & $3.96 \pm 0.66$ & $\mathbf{0 . 0 0 0 1}$ \\
Serum $\mathrm{HCO}_{3}{ }^{-}(\mathrm{mEq} / \mathrm{L})$ & $20.59 \pm 1.8$ & $23.1 \pm 3.5$ & $\mathbf{0 . 0 0 0 1}$
\end{tabular}

The data are reported in mean \pm standard deviation. Results of paired samples $t$-test.

Body weight, serum urea, creatinine, calcium, and potassium, and tear osmolarity of patients statistically decreased, and $\mathrm{HCO}_{3}{ }^{-}$increased significantly after $\mathrm{HD}(p=0.0001)$ (Table 2).

There was no significant correlation between pre-HD tear osmolarity and pre-HD serum osmolarity, glucose, urea, sodium, potassium, calcium, and bicarbonate levels and body weight $(p>0.05)$. Pre-HD tear osmolarity was statistically significantly correlated with pre-HD creatinine $(r=-0.366 p=0.016)$.

There was no significant correlation between post-HD tear osmolarity and post-HD serum urea, creatinine, sodium, potassium, calcium, and bicarbonate levels and body weight $(p>0.05)$. Post-HD tear osmolarity was statistically significantly correlated with post-HD glucose $(r=0.305$, $p=0.047$.

We also subgrouped patients according to the presence of diabetes mellitus type 2 (DM) and hypertension (HT). The difference regarding pre-HD and post-HD tear osmolarity between patients with or without DM was not statistically significant $(p>0.05)$ (Table 3$)$.

The difference regarding pre-HD tear osmolarity between patients with or without HT was not statistically significant $(p>0.05)$. But post-HD tear osmolarity of patients without HT was statistically significantly lower than patients with HT $(p=0.043)$ (Table 4$)$.

In correlation analysis, tear osmolarity difference was statistically significantly correlated with ultrafiltration, body weight difference, and creatinine difference but not with URR and $K t / V$ values. The $p$ values of correlation analysis are summarized in Table 5.

\section{Discussion}

The adequacy of $\mathrm{HD}$ is important for management of patients with ESRD. According to latest guidelines the minimally adequate dose of HD given 3 times per week to patients with $K_{r}$ less than $2 \mathrm{~mL} / \mathrm{min} / 1.73 \mathrm{~m}^{2}$ should be $\mathrm{sp} K t / V$ of 1.2 per dialysis. For treatment less than 5 hours, an alternative minimum dose is URR of $65 \%$ [3]. In our study HD achieved minimally adequate doses in 37 patients (86\%) but not in 6 patients.
TABLE 3: Effects of DM on pre-HD and post-HD tear osmolarity.

\begin{tabular}{lcccc}
\hline & DM & $n$ & Mean \pm SD & $p$ \\
\hline \multirow{2}{*}{ Pre-HD tear osmolarity } & DM (-) & 11 & $319.18 \pm 18.8$ & 0 \\
& DM (+) & 7 & $323.43 \pm 21.10$ & \\
\hline \multirow{2}{*}{ Post-HD tear osmolarity } & $\mathrm{DM}(-)$ & 11 & $302.10 \pm 14.06$ & \multirow{2}{*}{0.413} \\
& $\mathrm{DM}(+)$ & 7 & $308.86 \pm 20.23$ & \\
\hline
\end{tabular}

TABLE 4: Effects of HT on pre-HD and post-HD tear osmolarity.

\begin{tabular}{llccc}
\hline & HT & $n$ & Mean \pm SD & $p$ \\
\hline \multirow{2}{*}{ Pre-HD tear osmolarity } & HT (-) & 10 & $311.40 \pm 16.77$ & \multirow{2}{*}{0.482} \\
& HT (+) & 27 & $316.00 \pm 17.71$ & \\
\hline \multirow{2}{*}{ Post-HD tear osmolarity } & HT (-) & 10 & $295.50 \pm 11.21$ & \multirow{2}{0.043}{} \\
& HT (+) & 27 & $306.29 \pm 14.66$ & \\
\hline
\end{tabular}

TABLE 5: Correlation analysis.

\begin{tabular}{llc}
\hline \multirow{2}{*}{ Ultrafiltration } & & Tear osmolarity difference \\
\hline \multirow{2}{*}{ Body weight difference } & $r$ & -0.320 \\
& $p$ & $\mathbf{0 . 0 3 6}$ \\
\hline \multirow{2}{*}{ Creatinine difference } & $p$ & -0.365 \\
& $p$ & $\mathbf{0 . 0 1 6}$ \\
\hline \multirow{2}{*}{ URR } & $p$ & -0.426 \\
& $r$ & $\mathbf{0 . 0 0 4}$ \\
\multirow{2}{*}{$K t / V$} & $p$ & 0.057 \\
& $r$ & 0.718 \\
\hline
\end{tabular}

The results of Pearson's correlation analysis. $r$ : correlation coefficient; $p$ : statistical significance.

In an adequate hemodialysis serum sodium levels are determined as $135-145 \mathrm{mEq} / \mathrm{L}$, potassium $3-9 \mathrm{mEq} / \mathrm{L}$, calcium $7-12 \mathrm{mEq} / \mathrm{L}$, bicarbonate > $15 \mathrm{mEq} / \mathrm{L}$, creatinine < $12 \mathrm{mEq} / \mathrm{L}$, and albumin $>3 \mathrm{gr} / \mathrm{dL}$ [3]. In our study all of these biochemical markers were in these ranges.

In this study, we have evaluated tear osmolarity of patients with ESRD one minute before the beginning of $\mathrm{HD}$ and 30 minutes after the end of HD. We observed tear hyperosmolarity before $\mathrm{HD}$ and a significant reduction to normal levels after HD $(314.05 \pm 17.77 \mathrm{mOsm} / \mathrm{L}$ and $301.88 \pm$ $15.22 \mathrm{mOsm} / \mathrm{L}$, resp., $p<0.0001)$. Gilbard et al. indicate in two rabbit models for keratoconjunctivitis sicca that decreased tear volume or excessive evaporation is the major cause of tear hyperosmolarity [10]. Charlton et al. report tear hyperosmolarity, using freezing point depression method, (average $347 \mathrm{mOsm} / \mathrm{L}$, range $375-312 \mathrm{mOsm} / \mathrm{L}$ ) in $10 \mathrm{renal}$ dialysis patients in pre-HD and tested 5 of them immediately after completion of HD. They show a significant reduction in tear osmolarity after HD in all patients, correlating with our results. They speculate that, from the three principle solutes (sodium, glucose, and urea), urea is the only one that freely passes from serum to the tears and responsible for tear hyperosmolarity in renal dialysis patients [18]. 
In our study only one patient scored positively for dry eye before HD according to the McMonnies and Ho questionnaire and all patients gave the same answers to the questionnaire after HD. Similarly, Charlton et al. report that none of the renal dialysis subjects scored positively for dry eye. They speculate that, from the three principle solutes (sodium, glucose, and urea), urea is the only one that freely passes from serum to the tears and is responsible for tear hyperosmolarity in renal dialysis patients. According to them, hemodialysis patients remain asymptomatic for dry eye mainly because of the protective effects of urea in tears on the ocular surface [18].

In this study, we did not find any correlation between serum electrolyte levels and tear osmolarity both in pre-HD and post-HD periods. Aktaş et al. determined a prognostic importance of serum calcium levels for the ocular findings and symptoms in patients with ESRD [5]; however, we did not determine any correlation between pre-HD and post-HD calcium levels and tear osmolarity. Serum osmolarity also did not correlate with tear osmolarity.

There was no significant correlation between pre-HD tear osmolarity and pre-HD glucose and urea levels and body weight $(p>0.05)$. Pre-HD tear osmolarity was statistically significantly correlated with pre-HD creatinine $(r=-0.366$, $p=0.016)$. This is the first report denoting an association between tear osmolarity and creatinine according to our knowledge.

There was no significant correlation between post-HD tear osmolarity and post-HD serum urea and creatinine levels and body weight $(p>0.05)$. Post-HD tear osmolarity was statistically significantly correlated with post-HD glucose $(r=0.305 p=0.047)$.

In correlation analysis tear osmolarity difference was statistically significantly correlated with ultrafiltration $(r=$ $-0.320, p=0.036)$, body weight difference $(r=-0.365$, $p=0.016)$, and creatinine difference $(r=-0.426, p=0.004)$.

In another study among patients undergoing HD for ESRD, the incidence of reduced basal tear secretion and dry eye symptoms were reported to be higher in diabetic patients than in nondiabetics [19]. However, in our study, we have subgrouped patients according to the presence of DM and we did not determine any significant effects of diabetes in pre-HD or post-HD tear osmolarity. Post-HD tear osmolarity of patients with HT was statistically significantly higher than patients without HT ( $p=0.043)$. Studies with larger sample sizes are needed for exact relevance of these results.

Jung et al. reported decrease in tear break-up time (TBUT) and Schirmer's tests after HD [20]. We did not evaluated Schirmer's test and TBUT because of low patient compliance after HD.

This study has some limitations. As HD is a long lasting treatment method, patients may be divided into subgroups according to duration of HD. We have evaluated patients regardless of the duration of HD. Lack of Schirmer's test and TBUT are two other limitations. We could not do Schirmer's test and TBUT because of low patient compliance after HD. $\mathrm{K} t / V$ was $<1.2$ and URR was $<65 \%$ and $\mathrm{HD}$ was not adequate in only 6 patients. More patients are needed to assess the association between tear osmolarity and adequacy of HD.
In conclusion tear osmolarity is correlated with the serum creatinine levels in pre-HD period. In post-HD period, tear osmolarity is correlated with serum glucose levels. Tear osmolarity alteration induced by HD is correlated with body weight changes, creatinine alterations, and ultrafiltration. Therefore, HD corrects the volume and composition of the ocular fluid transiently.

\section{Disclosure}

None of the authors has a financial or personal relationship with other people or organizations that could inappropriately influence or bias the results.

\section{Conflict of Interests}

The authors declare that there is no conflict of interests regarding the publication of this paper.

\section{References}

[1] J. E. Flythe, G. C. Curhan, and S. M. Brunelli, "Shorter length dialysis sessions are associated with increased mortality, independent of body weight," Kidney International, vol. 83, no. 1, pp. 104-113, 2013.

[2] P. G. Blake, "Adequacy of dialysis revisited," Kidney International, vol. 63, no. 4, pp. 1587-1599, 2003.

[3] Hemodialysis Adequacy 2006 Work Group, "Clinical practice guidelines for hemodialysis adequacy, update 2006," American Journal of Kidney Diseases, vol. 48, pp. S2-S90, 2006.

[4] R. D. Evans and M. Rosner, "Ocular abnormalities associated with advanced kidney disease and hemodialysis," Seminars in Dialysis, vol. 18, no. 3, pp. 252-257, 2005.

[5] S. Aktaş, H. M. Sağdık, H. Aktaş et al., "Tear function in patients with chronic renal failure undergoing hemodialysis," Renal Failure, vol. 37, no. 2, pp. 245-248, 2015.

[6] A. Akinci, N. Cakar, N. Kara, and N. Uncu, "Ocular findings in children with chronic renal failure," Cornea, vol. 28, no. 1, pp. 5-6, 2009.

[7] M. R. Manaviat, M. Rashidi, M. Afkhami-Ardekani, and M. R. Shoja, "Prevalence of dry eye syndrome and diabetic retinopathy in type 2 diabetic patients," BMC Ophthalmology, vol. 8, article 10, 2008.

[8] International Dry Eye WorkShop, "The definition and classification of dry eye disease: report of the Definition and Classification Subcommittee of the International Dry Eye WorkShop (2007)," The Ocular Surface, vol. 5, no. 2, pp. 75-92, 2007.

[9] M. A. Lemp, A. J. Bron, C. Baudouin et al., "Tear osmolarity in the diagnosis and management of dry eye disease," The American Journal of Ophthalmology, vol. 151, no. 5, pp. 792-798, 2011.

[10] J. P. Gilbard, S. R. Rossi, K. L. Gray, L. A. Hanninen, and K. R. Kenyon, "Tear film osmolarity and ocular surface disease in two rabbit models for keratoconjunctivitis sicca," Investigative Ophthalmology and Visual Science, vol. 29, no. 3, pp. 374-378, 1988.

[11] J. Murube, “Tear osmolarity," Ocular Surface, vol. 4, no. 2, pp. 62-73, 2006. 
[12] U. Stahl, M. Willcox, and F. Stapleton, "Osmolality and tear film dynamics," Clinical \& Experimental Optometry, vol. 95, no. 1, pp. 3-11, 2012.

[13] A. T. Azar, "Adaptive network based on fuzzy inference system for equilibrated urea concentration prediction," Computer Methods and Programs in Biomedicine, vol. 111, no. 3, pp. 578591, 2013.

[14] J. H. Thaysen and N. A. Thorn, "Excretion of urea, sodium, potassium and chloride in human tears," The American Journal of Physiology, vol. 178, no. 1, pp. 160-164, 1954.

[15] L. I. Worthley, M. Guerin, and R. W. Pain, "For calculating osmolality, the simplest formula is the best," Anaesthesia \& Intensive Care, vol. 15, no. 2, pp. 199-202, 1987.

[16] J. T. Daugirdas, "Second generation logarithmic estimates of single-pool variable volume Kt/V: an analysis of error," Journal of the American Society of Nephrology, vol. 4, no. 5, pp. 12051213, 1993.

[17] C. McMonnies, A. Ho, and D. Wakefield, "Optimum dry eye classification using questionnaire responses," in Lacrimal Gland, Tear Film, and Dry Eye Syndromes 2, vol. 438 of Advances in Experimental Medicine and Biology, pp. 835-838, Springer, 1998.

[18] J. F. Charlton, I. R. Schwab, and R. Stuchell, “Tear hyperosmolarity in renal dialysis patients asymptomatic for dry eye," Cornea, vol. 15, no. 4, pp. 335-339, 1996.

[19] M. Nakata, Y. Okada, H. Kobata et al., "Diabetes mellitus suppresses hemodialysis-induced increases in tear fluid secretion," BMC Research Notes, vol. 7, article 78, 2014.

[20] J. W. Jung, M. H. Yoon, S. W. Lee, and H. S. Chin, "Effect of hemodialysis (HD) on intraocular pressure, ocular surface, and macular change in patients with chronic renal failure. Effect of hemodialysis on the ophthalmologic findings," Graefe's Archive for Clinical and Experimental Ophthalmology, vol. 251, no. 1, pp. 153-162, 2013. 


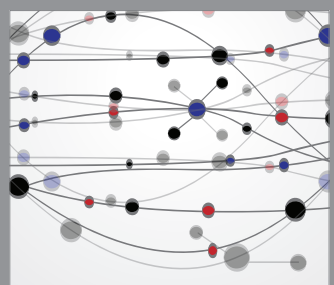

The Scientific World Journal
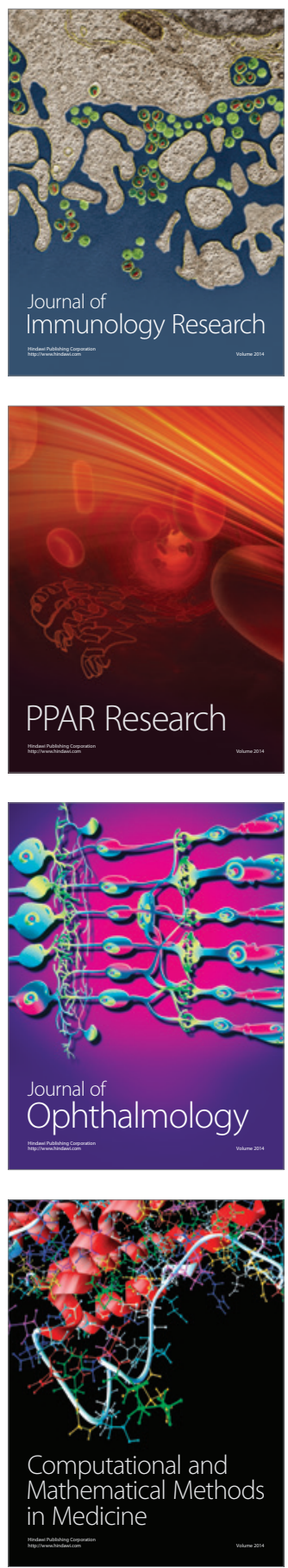

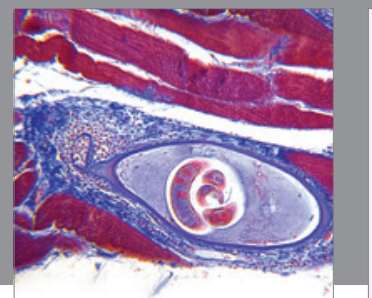

Gastroenterology

Research and Practice
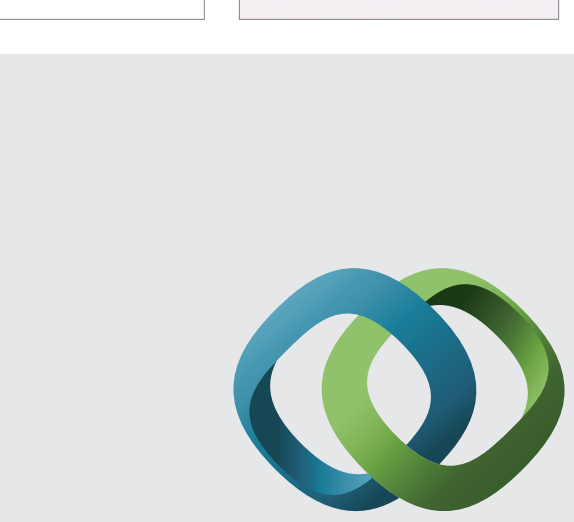

\section{Hindawi}

Submit your manuscripts at

http://www.hindawi.com
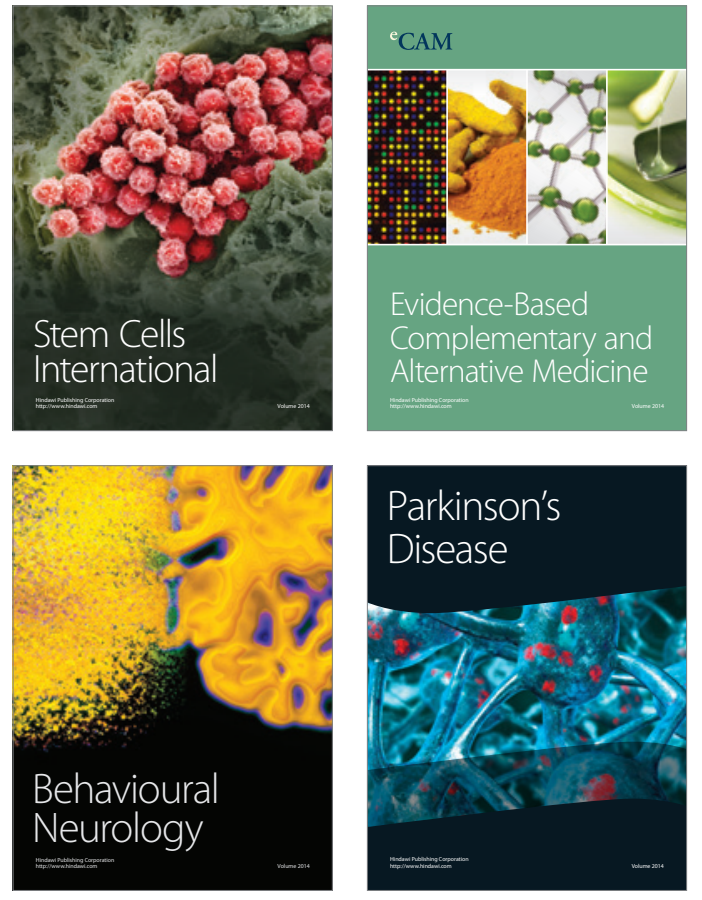
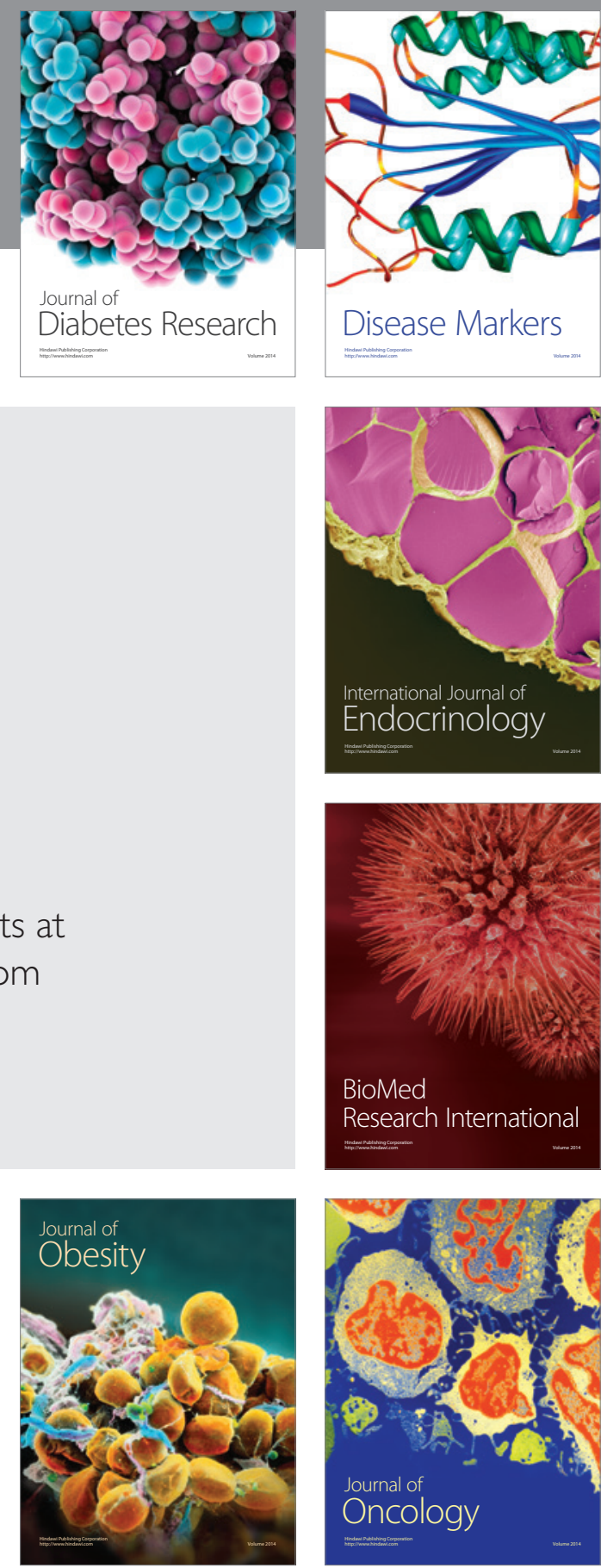

Disease Markers
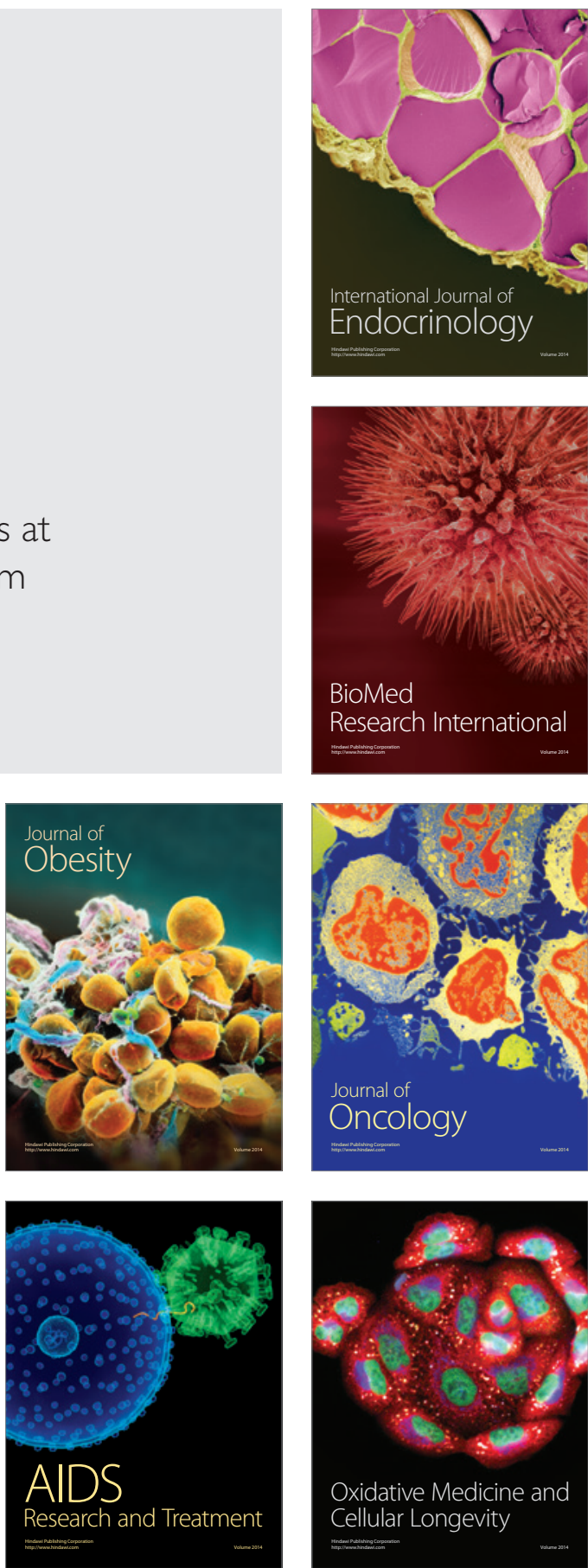